\title{
COUNTEREXAMPLE TO THE LU QI-KENG CONJECTURE
}

\author{
HAROLD P. BOAS ${ }^{1}$
}

\begin{abstract}
There exists a smooth bounded strongly pseudoconvex domain in $\mathbf{C}^{2}$, smoothly equivalent to the ball, whose Bergman kernel function has zeroes.
\end{abstract}

The Bergman kernel function of the ball in $\mathbf{C}^{n}$ has no zeroes. Is it true that for every bounded domain the Bergman kernel function has no zeroes? Lu Qi-Keng [4] raised this question in 1966. Subsequent authors, attributing a positive opinion to him, called the affirmative answer "the Lu Qi-Keng conjecture".

The conjecture turned out to be too bold, for Skwarczyński [7] and Rosenthal [6] showed that it fails for annuli in the plane, and Greene and Krantz [2] observed that this counterexample lifts to higher dimensions. Suita and Yamada [8] clarified the situation by proving that the smoothly bounded planar domains for which the Lu Qi-Keng conjecture holds are precisely the simply connected ones.

It has remained open whether the $\mathrm{Lu} \mathrm{Qi}$-Keng conjecture is valid for topologically trivial domains in higher dimensions also [3]. The following simple example shows that it is not.

THEOREM. There exists a smooth bounded strongly pseudoconvex domain in $\mathbf{C}^{2}$ whose closure is diffeomorphic to the ball and whose Bergman kernel function has zeroes.

PROOF. First consider the unbounded logarithmically convex complete Reinhardt domain $D$ consisting of the points $(z, w)$ in $\mathbf{C}^{2}$ for which $|w|<(1+|z|)^{-1}$. Every function holomorphic in $D$ has a power series expansion converging in the whole of $D$. Integration in polar coordinates shows that an orthogonal basis for the space of square-integrable holomorphic functions in $D$ consists of the monomials $z^{j} w^{k}$ for which $j<k$. Thus every square-integrable holomorphic function in $D$ vanishes when $w=0$, and in particular the Bergman kernel function for $D$ has zeroes.

Let $D_{m}$ denote the intersection of $D$ with the ball of radius $m$ centered at the origin. By Ramadanov's theorem [5] the Bergman kernel for $D_{m}$ converges uniformly on compact sets to the Bergman kernel for $D$ as $m \rightarrow \infty$. (Ramadanov gave the theorem for bounded domains in $\mathbf{C}^{1}$ and stated only convergence in one variable with the other fixed, but the more general result requires only a slight modification of his proof.) By Hurwitz's theorem the Bergman kernel for $D_{m}$ has zeroes when $m$ is large enough.

Such a $D_{m}$ is a bounded topologically trivial domain of holomorphy for which the Lu Qi-Keng conjecture fails. The domain described in the theorem is obtained by

Received by the editors September 3, 1985.

1980 Mathematics Subject Classification. Primary 32H10.

${ }^{1}$ Partially supported by National Science Foundation grant no. DMS-8501758. 
approximating $D_{m}$ from within by smooth strongly logarithmically convex complete Reinhardt domains.

REMARKS. (1) Already from examples of Wiegerinck [9] and Azukawa [1] one can construct a bounded contractible domain in $\mathbf{C}^{2}$ whose Bergman kernel has zeroes; but it is not a domain of holomorphy.

(2) In [2] Greene and Krantz proved that in the $C^{\infty}$ topology on the class of smooth bounded strongly pseudoconvex domains the set of domains for which the Bergman kernel function is bounded away from zero is open, while the set of domains for which the Bergman kernel function has no zeroes is closed. The above example implies that the second set is strictly larger than the first.

(3) I have learned that David Catlin found a counterexample similar to mine, but he did not publish it.

\section{BIBLIOGRAPHY}

1. K. Azukawa, Square-integrable holomorphic functions on a circular domain in $\mathbf{C}^{n}$, Tôhoku Math J. 37 (1985), 15-26.

2. R. E. Greene and S. G. Krantz, Stability properties of the Bergman kernel and curvature properties of bounded domains, Recent Developments in Several Complex Variables, Princeton Univ. Press, Princeton, N. J., 1981.

3. S. G. Krantz, Function theory of several complex variables, Wiley, New York, 1982.

4. Lu Qi-Keng, On Kaehler manifolds with constant curvature, Chinese Math. 8 (1966), 283-298; English transl. of Acta Math. Sinica 16 (1966), 269-281.

5. I. Ramadanov, Sur une propriété de la fonction de Bergman, C. R. Acad. Bulgare Sci. 20 (1967), 759-762.

6. P. Rosenthal, On the zeroes of the Bergman function in doubly-connected domains, Proc. Amer. Math. Soc. 21 (1969), 33-35.

7. M. Skwarczyński, The invariant distance in the theory of pseudoconformal transformations and the Lu Qi-Keng conjecture, Proc. Amer. Math. Soc. 22 (1969), 305-310.

8. N. Suita and A. Yamada, On the Lu Qi-Keng conjecture, Proc. Amer. Math. Soc. 59 (1976), 222-224.

9. J. J. O. O. Wiegerinck, Domains with finite dimensional Bergman space, Math. Z. 187 (1984), 559-562.

Department of Mathematics, Texas a\&M University, College Station, TEXAS 77843 